\title{
Uric Acid Induces Cardiomyocyte Apoptosis via Activation of Calpain-1 and Endoplasmic Reticulum Stress
}

\author{
Meiling Yan ${ }^{a}$ Kankai Chen ${ }^{a} \quad$ Li He $^{b}$ Shuai Lia Dong Huanga Jingbo Lia \\ aDepartment of Cardiology, Shanghai Jiao Tong University Affiliated Sixth People's Hospital, Shanghai, \\ 'Department of Nephrology, Shanghai Jiao Tong University Affiliated Sixth People's Hospital, Shanghai, \\ China
}

\section{Key Words}

Uric acid • Calpain • Endoplasmic reticulum stress $•$ Apoptosis

\begin{abstract}
Background/Aims: Hyperuricemia is associated with an increased risk for multiple cardiovascular diseases, but the underlying mechanisms remain largely elusive. Calpain-1 is a protease that is implicated in several pathological conditions that affect the heart. The aim of this current study was to test the effects of uric acid (UA) on cardiomyocyte survival and cardiac function and to investigate the role of calpain-1 in the UA-induced effects in the heart and their underlying mechanisms. Methods: In vivo, hyperuricemia was induced by oxonic acid (OA) administration in Sprague-Dawley rats for 16 weeks; TUNEL staining was used to identify apoptotic cells. Left ventricular (LV) sections were stained with Sirius Red to evaluate interstitial fibrosis. Cardiac catheterization was performed to evaluate cardiac function. In vitro, cultured H9c2 cells were incubated with different UA concentrations. MTT assays and flow cytometry were used to evaluate cell viability and apoptosis. All related gene expression levels were analyzed by quantitative real-time PCR (qRT-PCR), and all protein expression levels were analyzed by western blotting. Results: Hyperuricemia induction in vivo resulted in cellular apoptosis, interstitial fibrosis and diastolic dysfunction in the rat hearts, as well as increased activation of calpain-1 and endoplasmic reticulum (ER) stress, while allopurinol treatment mitigated the above changes. UA administration in vitro increased apoptosis and decreased H9c2 cell viability in a dose-dependent manner. Increased activation of calpain-1 and ER stress was also observed in the groups with high UA levels. Calpain-1 siRNA and the calpain inhibitor CI-III alleviated UA-induced ER stress and apoptosis, while inhibiting ER stress by tauroursodeoxycholic acid (TUDCA) mitigated UA-induced apoptosis without affecting calpain-1 expression or activity. Conclusions: These findings suggest that UA induces cardiomyocyte apoptosis through activation of calpain- 1 and ER stress. These results may
\end{abstract}

M. Yan and K. Chen contributed equally to this work.

Jingbo Li

KARGER
Department of Cardiology, Shanghai Jiao Tong University Affiliated Sixth People's Hospital, 600 Yishan Road, Shanghai (China)

Tel. +86-21-24058334, Fax +86-21-64701932, E-Mail lijb@sjtu.edu.cn 
provide new insights into the mechanisms of hyperuricemia-associated cardiovascular risks and hopefully identify new treatment targets.

(C) 2018 The Author(s)

Published by S. Karger AG, Basel

\section{Introduction}

Uric acid (UA) is the end product of purine metabolism in humans and higher primates [1]. Abnormally high level of UA, also known as hyperuricemia, is not only important in the pathophysiological process of gout, but also a risk factor for multiple cardiovascular diseases, such as coronary heart disease, hypertension, atrial fibrillation, and heart failure [2-5]. These adverse effects of UA on the cardiovascular system may be related to the role of UA in several pathological processes [6-8]. However, the underlying mechanisms are not fully understood.

Calpains are a class of calcium-activated non-lysosomal cysteine proteases that serve as proteolytic units for cellular homeostasis [9]. Under basal conditions, calpain is predominantly located in the cytosol and translocates to the membrane after activation [10]. To date, 15 calpain isoforms have been reported. Among these isoforms, two major isoforms, calpain-1 and calpain-2, are ubiquitously expressed in several systems including the heart, whereas the other isoforms have more limited tissue distribution [11]. In cardiovascular system, calpains play significant roles in several pathological conditions including hypertension [12], myocardial infarction [13], and heart failure [14]. However, the role of calpains in hyperuricemia-induced effects on the cardiovascular system has not been studied.

The aim of the present study was to test the effects of UA on myocardial survival and cardiac function, as well as the involvement of calpains in UA induced cell death and their underlying mechanisms. To achieve these goals, an in vivo hyperuricemia model was induced in rats, and UA was administered to H9c2 cells in vitro.

\section{Materials and Methods}

\section{Chemicals}

UA, tauroursodeoxycholic acid (TUDCA), the calpain inhibitor CI-III, allopurinol, and 3-(4, 5-dimethylthiazol-2-yl)-2, 5-diphenyltetrazolium bromide (MTT) were purchased from Sigma (St. Louis, MO, USA). The Annexin V-FITC Apoptosis Detection kit was purchased from Invitrogen (Carlsbad, CA, USA). Primary antibodies against calpain-1, calpain-2, total-PRKR-like ER kinase (PERK), CHOP, Bax, Bcl and GAPDH were purchased from Cell Signaling Technology (Boston, MA, USA). Antibodies against GRP78 and $\alpha$-actinin were purchased from Abcam (Cambridge, UK). Antibodies against calpain-1 and phosphorylated PERK was purchased from Santa Cruz Biotechnology (Santa Cruz, CA, USA). HRP-linked secondary antibodies against rabbit IgG and mouse IgG were purchased from Cell Signaling Technology (Boston, MA, USA). Alexa Fluor 555-conjugated goat anti-mouse and Alexa Fluor 647-conjugated goat anti-rabbit secondary antibodies were purchased from Invitrogen (Carlsbad, CA, USA). Penicillin, streptomycin, Dulbecco's modified Eagle's medium (DMEM), and fetal bovine serum (FBS) were obtained from Gibco (Grand Island, NY, USA).

\section{Animals and experimental protocols}

A total of 60 male Sprague-Dawley rats (6 weeks old), weighing between 200 and 220 g (SIPPR-BK Laboratory Animal Co. Ltd., Shanghai, China) were used in the present study. All rats were housed at a controlled temperature $\left(25^{\circ} \mathrm{C}\right)$ under a 12-h light/dark cycle with ad libitum access to food and water. All the animal maintenance and experimental procedures were in compliance with the Guide for the Care and Use of Laboratory Animals published by the National Institutes of Health and approved by the Institutional Animal Care and Use Committee (IACUC) of Shanghai Jiao Tong University Affiliated Sixth People's Hospital (ethical approval code No. DWLL2017-0272).

After 1 week of acclimation, the rats were randomly divided into four groups: (1) the control group $(n=15)$ was gavaged with vehicle $(0.9 \%$ saline); $(2)$ the allopurinol group $(n=15)$ was gavaged with allopurinol (10 mg/kg/day); (3) the oxonic acid (OA) group $(\mathrm{n}=15)$ was gavaged with $\mathrm{OA}(750 \mathrm{mg} / \mathrm{kg} / \mathrm{day})$; 


\section{Cellular Physiology Cell Physiol Biochem 2018;45:2122-2135 \\ \begin{tabular}{ll|l} 
DOI: 10.1159/000488048 & and Biochemistry & $\begin{array}{l}\text { ( 2018 The Author(s). Published by S. Karger AG, Basel } \\
\text { www.karger.com/cpb }\end{array}$
\end{tabular}}

Yan et al.: UA Induces Apoptosis in Cardiomyocytes via Calpain-1

and (4) the OA+allopurinol group ( $\mathrm{n}=15)$ was gavaged with $\mathrm{OA}(750 \mathrm{mg} / \mathrm{kg} / \mathrm{day})$ and allopurinol (10 mg/kg/ day). After 16 weeks of gavage administration, the rats were housed in metabolic cages for urine collection over $24 \mathrm{~h}$. The rats were then anesthetized with pentobarbital sodium ( $50 \mathrm{mg} / \mathrm{kg}$ ), and catheterization was performed to assess hemodynamic changes. The rats were sacrificed with pentobarbital sodium (150 mg/ $\mathrm{kg}$ ) overdose, and blood and heart tissues were harvested for further assessment.

\section{Cell culture and treatments}

The H9c2 embryonal rat heart-derived cell line was obtained from the American Type Culture Collection (ATCC, Manassas, VA, USA). H9c2 cells were cultured in DMEM supplemented with 10\% FBS, $100 \mathrm{U} / \mathrm{ml}$ penicillin and $100 \mu \mathrm{g} / \mathrm{ml}$ streptomycin in a humidified incubator at $37^{\circ} \mathrm{C}$ with $5 \% \mathrm{CO}_{2}$. The cells were treated with final UA concentrations of 50,100, 200 and $400 \mu \mathrm{M}$. To determine the role of endoplasmic reticulum (ER) stress in UA-induced apoptosis, the ER stress inhibitor tauroursodeoxycholic acid (TUDCA) $(100 \mu \mathrm{M})$ was added $30 \mathrm{~min}$ prior to the UA addition. The role of calpain on UA-induced apoptosis was studied by preincubating the cells with the calpain inhibitor CI-III $(10 \mu \mathrm{M})$ for $30 \mathrm{~min}$.

To knockdown calpain-1, H9c2 cells were transfected with small interfering RNA (siRNA) against rat calpain-1, purchased from Santa Cruz Biotechnology (Santa Cruz, CA, USA, sc-60099), and a negative control siRNA (Santa Cruz, CA, USA, siRNAsc-37007) was used as a control. Transfection was performed using HiPerFect transfection reagent (Qiagen, Hilden, Germany) according to manufacturer's instructions. Further experiments were performed after $48 \mathrm{~h}$ of siRNA infection.

\section{MTT assay for cell viability}

Cell viability was measured by the 3-(4, 5-dimethylthiazol-2-yl)-2, 5-diphenyl tetrazolium bromide (MTT) assay. H9c2 cells were seeded onto 96-well plates at a density of $1 \times 10^{4}$ cells per well and were incubated at $37^{\circ} \mathrm{C}$ for $24 \mathrm{~h}$. After the treatments, the culture medium was discarded, $150 \mu \mathrm{l}$ of MTT solution (final concentration of $0.5 \mathrm{mg} / \mathrm{ml}$ ) was added to each well, and the cells were incubated for $4 \mathrm{~h}$ at $37{ }^{\circ} \mathrm{C}$. Then, the MTT solution was removed, and $150 \mu \mathrm{l}$ of dimethylsulfoxide was added to dissolve the formazan crystals. The absorbance at $490 \mathrm{~nm}$ was read on a microplate reader (BioTek, Winooski, VT, USA). The absorbance was used to measure cell viability, and the absorbance of the treatment groups was normalized to that of the control group, which was considered $100 \%$ viable.

\section{Apoptosis assay using flow cytometry}

The H9c2 cells were stained using an Annexin V/PI cell apoptosis kit (Invitrogen, Carlsbad, CA, USA) according to the manufacturer's instructions. Briefly, H9c2 cells were harvested after the incubation period and washed in cold phosphate-buffered saline (PBS). The cells were centrifuged and resuspended in $1 \times$ annexin-binding buffer. Then, the cells were counted and diluted to $1 \times 10^{6}$ cells $/ \mathrm{mL}$ in $1 \times$ annexin-binding buffer. Each $100 \mu \mathrm{L}$ of cell suspension was incubated at room temperature for 15 minutes, followed by the addition of $5 \mu \mathrm{L}$ of Annexin V and $1 \mu \mathrm{L}$ of PI working solution. After the incubation period, $400 \mu \mathrm{L}$ of $1 \times$ annexin-binding buffer was added to the samples. Samples were assayed within $1 \mathrm{~h}$ using a flow cytometer (BD Biosciences, Franklin lakes, NJ, USA), recording ten thousand events. The apoptosis ratio was calculated as the number of early apoptotic cells relative to the total number of cells.

\section{Calpain activity assay}

Calpain enzymatic activity was determined with a calpain activity assay kit (BioVision, Milpitas, CA, USA) according to the manufacturer's protocol. Briefly, H9c2 cells and heart tissues were resuspended in extraction buffer. Then, each sample was incubated on ice for $20 \mathrm{~min}$. The cell lysate was centrifuged for $1 \mathrm{~min}(10,000 \mathrm{~g})$, and the supernatant was transferred to a fresh tube and stored on ice. After protein concentration determination, $50 \mu \mathrm{g}$ of lysate was diluted in $85 \mu \mathrm{l}$ of extraction buffer. After the addition of $10 \mu \mathrm{l}$ of $10 \times$ reaction buffer and $5 \mu \mathrm{l}$ of calpain substrate, each sample was incubated at $37^{\circ} \mathrm{C}$ for $1 \mathrm{~h}$ in the dark. Calpain activity was measured by a luminescence spectrometer (Perkin-Elmer, Shelton, CT, USA) equipped with a 400-nm excitation filter and 505-nm emission filter. For each analysis, calpain activity was normalized to the control activity value.

TUNEL staining

The apoptosis rate was measured using the commercially available TUNEL assay kit (In Situ Cell Death Detection kit; Roche Diagnostics GmbH, Mannheim, Germany), according to the manufacturer's protocol. 


\section{Cellular Physiology Cell Physiol Biochem 2018;45:2122-2135

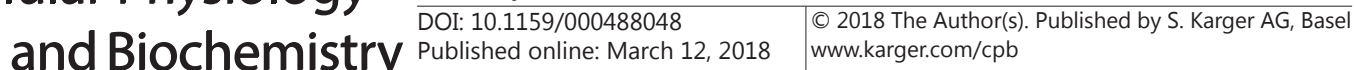

Yan et al.: UA Induces Apoptosis in Cardiomyocytes via Calpain-1

Briefly, consecutive 4- $\mu$ m left ventricular (LV) sections were deparaffinized with xylene and rehydrated in graded alcohol. Then, the tissue sections were incubated with proteinase $\mathrm{K}$ at $37^{\circ} \mathrm{C}$ for $30 \mathrm{~min}$. After rinsing the sections twice with PBS, the sections were incubated with the prepared TUNEL reaction mixture at $37^{\circ} \mathrm{C}$ in the humidified atmosphere of a dark chamber for $60 \mathrm{~min}$, and then, the sample slices were soaked in the stop buffer and gently washed with PBS. Converter-POD (50 $\mu \mathrm{l})$ was added to each section, and the sections were incubated in a humidified chamber for $30 \mathrm{~min}$ at $37^{\circ} \mathrm{C}$. After washing with PBS, the sections were incubated with $100 \mu \mathrm{l}$ of 3, 3'-diaminobenzidine (DAB) for $10 \mathrm{~min}$ at $25^{\circ} \mathrm{C}$. Apoptosis was visualized under a light microscope (Olympus BX60, Japan) as brown staining in the nuclei. Ten random fields were counted per section. The percentage of TUNEL-positive cardiomyocytes was calculated as follows: number of apoptotic cells / total number of cells $\times 100 \%$.

\section{Sirius Red staining for collagen}

The mouse hearts were fixed in 4\% formaldehyde and embedded in paraffin, and 4- $\mu \mathrm{m} \mathrm{LV} \mathrm{sections}$ were stained with aqueous saturated picric acid solution containing 0.1\% Sirius Red (Sigma-Aldrich, St. Louis, MO) for $10 \mathrm{~min}$ at room temperature. Then, the sections were dehydrated in $100 \%$ alcohol and cleared in xylene. Collagen fibers were visualized through red staining under a light microscope (Olympus BX60, Japan). Finally, ten random fields were recorded per section and then analyzed on Image-Pro Plus software 6.0 (Media Cybernetics, Silver Spring, MD, USA).

\section{Confocal imaging}

Fresh LV tissue samples were fixed in $4 \%$ formaldehyde and embedded in tissue freezing medium (optimal cutting temperature (OCT) compound); the embedded tissues were then frozen at $-80{ }^{\circ} \mathrm{C}$. Cryosections (6- $\mu \mathrm{m}$ thick) were prepared using a Leica cryomicrotome for immunohistochemistry. Sections were fixed in $4 \%$ formaldehyde, permeabilized with $0.4 \%$ Triton X-100, and washed with PBS. Apoptotic cell death was detected by TUNEL staining according to the manufacturer's protocol. Then, the sections were blocked with $10 \%$ normal donkey serum for $1 \mathrm{~h}$ at room temperature and incubated overnight at $4{ }^{\circ} \mathrm{C}$ with the following primary antibodies: rabbit anti- $\alpha$-actinin antibody (1:200, Abcam, ab137346) and mouse anticalpain-1 antibody (1:200, Santa Cruz Biotechnology, sc-390677). After the sections were washed with PBS, Alexa Fluor 647-labeled anti-rabbit IgG (1:200, Invitrogen, A21245) and Alexa Fluor 555-labeled anti-mouse IgG (1:200, Invitrogen, A21422) were used as secondary antibodies and were incubated with the sections for $1 \mathrm{~h}$ at room temperature. Nuclei were stained with 4, 6-diamidino-2-phenylindole (Molecular Probes, Eugene, OR, USA). The fluorescence staining was visualized using laser scanning confocal microscopy (Zeiss, Oberkochen, Germany).

\section{Cardiac functional assessment}

The right carotid artery was cannulated using a 1.6F pressure catheter (Transonic Scisense, Inc., London, $\mathrm{ON}$, Canada) to measure the hemodynamic parameters. The catheter was passed retrogradely into the left ventricle, and LV pressure tracings were digitized using a PowerLab Physiological Recorder (ADInstruments Pty, Ltd., Bella Vista, Australia) and stored for later analysis. The heart rate, LV end-systolic pressure (LVESP), $\mathrm{LV}$ end-diastolic pressure (LVEDP) and maximal rate of the increase/decrease of LV pressure $\left( \pm \mathrm{dP} / \mathrm{dt}_{\text {max }}\right)$ were analyzed in a blinded-manner using LabChart software, version 8 (ADInstruments Pty Ltd., Oxford, UK).

\section{Western blot analysis}

Total protein was collected from freshly dissected rat hearts and cell lysates. The protein concentration was determined using a BCA kit (Pierce, Rockford, IL, USA). Equal amounts of protein were electrophoresed in 8-12\% SDS-polyacrylamide gels and then transferred to polyvinylidene fluoride (PVDF) membranes (Millipore, Bedford, MA, USA). The membranes were blocked for $2 \mathrm{~h}$ with 5\% milk at room temperature and then incubated overnight at $4{ }^{\circ} \mathrm{C}$ with the following primary antibodies: calpain-1 (1:1000; Cell Signaling Technology, \#2556), calpain-2 (1:1000; Cell Signaling Technology, \#2539), total-PERK (1:1000; Cell Signaling Technology, \#3192), CHOP (1:500; Cell Signaling Technology, \#2895), Bax (1:1000; Cell Signaling Technology, \#2772), Bcl (1:1000; Cell Signaling Technology, \#2870), GAPDH (1:10000; Cell Signaling Technology, \#2118), GRP78 (1:10000, Abcam, ab21685) and phosphorylated PERK (1:500, Santa Cruz Biotechnology, sc-32577). After three washes with Tris-buffered saline containing 0.05\% Tween 20 
(TBST) for $10 \mathrm{~min}$, the membranes were incubated with horseradish peroxidase-conjugated secondary antibodies for $1 \mathrm{~h}$ and finally examined by an electrochemiluminescence (ECL) reagent (Millipore, Billerica, MA, USA). Images were captured using an image reader LAS-4000 mini system (Fujifilm, Tokyo, Japan) and quantified using Gel Pro Analyzer software (Media Cybernetics, Silver Spring, MD, USA).

\section{Reverse transcription and real-time PCR}

LV myocardial tissues were homogenized, and total RNA was isolated using TRIzol reagent (Invitrogen, Carlsbad, CA) according to the manufacturer's instructions. The RNA was treated with gDNA Eraser to remove genomic DNA contamination. Total RNA was then reverse-transcribed into cDNA using the PrimeScript ${ }^{\mathrm{TM}}$ RT reagent kit (Takara, Shiga, Japan), and the target genes were amplified using SYBR Premix Ex Taq II (Takara, Shiga, Japan). Amplification was performed with a real-time PCR system 7500 (Applied Biosystems) using the following conditions: initial denaturation at $95^{\circ} \mathrm{C}$ for $30 \mathrm{~s}$, followed by 40 cycles performed at $95{ }^{\circ} \mathrm{C}$ for $5 \mathrm{~s}$ and $60^{\circ} \mathrm{C}$ for $34 \mathrm{~s}$. The primers ( $5^{\prime}$ to $\left.3^{\prime}\right)$ for target genes were as follows: collagen-1 (F) GCAGATTGAGAACATCCGCAG and (R) GCAGCCTTGGTTAGGGTCG; CTGF (F) TTCCCGAGAAGGGTCAAGI and (R) GGCAGTTGGCT CGCATCA; TGF- $\beta$ (F) GGCGGTGCTCGCTTIA and (R) ATTGCGTTGTTGCGGTCC; and GAPDH (F) GGTGCTGAGTATGTIGGAG and (R) TTGCTGACAATCTTGAGGGAG. The fold change in gene expression induction or repression, as determined by real-time RT-PCR, was calculated using the $2^{-\Delta \Delta C t}$ formula after adjusting for GAPDH expression.

\section{Statistical analysis}

All data are expressed as the means \pm standard deviation (SD) and were analyzed with SPSS 18.0 statistical software (SPSS Inc., Chicago, IL, USA). Differences between two groups were compared by unpaired Student's t-tests. Differences among multiple groups were evaluated using one-way analysis of variance (ANOVA), followed by the Student-Newman-Keuls test. A value of $P<0.05$ was considered statistically significant.

\section{Results}

Hyperuricemia induced diastolic dysfunction, interstitial fibrosis and myocardial apoptosis in rat hearts

Hyperuricemia was induced by OA gavage administration for 16 weeks. As shown in Table 1, the OA administration significantly increased the plasma and urine UA levels but did not significantly influence serum creatinine levels. Cardiac catheterization was performed to test systolic and diastolic function of rat hearts. As shown in Table 2 , hyperuricemia induction resulted in impaired diastolic function, as evidenced by the decreased $-\mathrm{dP} / \mathrm{dt}_{\max }$ and increased LVEDP, and preserved systolic function. Furthermore, hyperuricemia-induced LV interstitial fibrosis, as evidenced by the markedly increased number

Table 1. Body weight and UA and creatinine concentrations at the end of the study. Data are expressed as the means \pm SD. ALLO, allopurinol; OA, oxonic acid; " $\mathrm{P}<0.05$ compared with the control group; ${ }^{*} \mathrm{P}<0.05$ compared with the OA group

\begin{tabular}{lcccc}
\hline & Control & ALLO & OA & OA+ALLO \\
\hline Body weight $(\mathrm{g})$ & $540 \pm 19$ & $546 \pm 25$ & $520 \pm 16$ & $542 \pm 21$ \\
Serum UA $(\mu \mathrm{M})$ & $69.3 \pm 8.4$ & $70.4 \pm 8.1$ & $176.2 \pm 19.1^{*}$ & $83.9 \pm 9.8^{\#}$ \\
Urinary UA $(\mu \mathrm{M} / 24 \mathrm{~h})$ & $13.1 \pm 1.5$ & $12.3 \pm 0.8$ & $31.4 \pm 2.4^{*}$ & $16.7 \pm 1.4^{\#}$ \\
serum creatinine $(\mu \mathrm{M})$ & $37.1 \pm 3.9$ & $38.3 \pm 4.2$ & $42.6 \pm 4.4$ & $39.3 \pm 4.0$ \\
\hline
\end{tabular}

Table 2. Cardiac function assessment at the end of the study. Data are expressed as the means \pm SD. ALLO, allopurinol; OA, oxonic acid; LVSDP, left ventricular end-systolic pressure LVEDP, left ventricular end-diastolic pressure; $+\mathrm{dP} / \mathrm{dt}_{\text {max }}$ maximal rate of the increase in left ventricular pressure; $-\mathrm{dP} / \mathrm{dt}_{\text {max }}$, maximal rate of the decrease in left ventricular pressure; " $\mathrm{P}<0.05$ compared with the control group; ${ }^{\mathrm{P}} \mathrm{P} 0.05$ compared with the OA group

\begin{tabular}{lcccc}
\hline & Control & ALLO & OA & OA+ALLO \\
\hline Heart rate (bpm) & $384 \pm 36$ & $386 \pm 41$ & $378 \pm 39$ & $378 \pm 34$ \\
LVSDP (mmHg) & $75 \pm 8.1$ & $78 \pm 8.5$ & $82 \pm 8.8$ & $74 \pm 7.2$ \\
LVEDP (mmHg) & $4.1 \pm 0.61$ & $4.1 \pm 0.65$ & $6.3 \pm 0.76^{*}$ & $4.2 \pm 0.55^{\#}$ \\
$+\mathrm{dP} / \mathrm{dt}_{\max }(\mathrm{mmHg} / \mathrm{s})$ & $8579 \pm 625$ & $8813 \pm 715$ & $8450 \pm 581$ & $8670 \pm 659$ \\
-dP/dt $\max (\mathrm{mmHg} / \mathrm{s})$ & $4936 \pm 503$ & $4950 \pm 559$ & $3567 \pm 371^{*}$ & $4885 \pm 450^{\#}$ \\
\hline
\end{tabular}


Fig. 1. Hyperuricemia induced interstitial fibrosis in the rat myocardium. (A) Representative heart images stained for fibrotic tissue deposition by Sirius Red staining (collagen is stained bright red) in each group (magnification, 400×). (B) Quantification of the cardiac fibrosis area in each group. (C) mRNA expression of fibrosis markers (Collagen-1, CTGF and TGF- $\beta$ ) in the myocardial tissues. Data are expressed as the means $\pm \mathrm{SD}$. ${ }^{*} \mathrm{P}<0.05$ compared with the control group; ${ }^{\#} \mathrm{P}<0.05$ compared with the OA group.

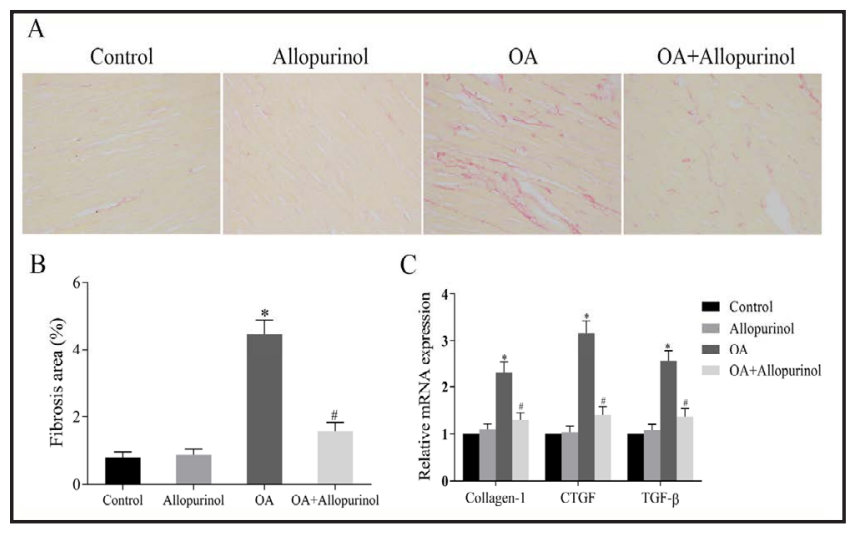

Fig. 2. Hyperuricemia increased apoptosis in the rat myocardium. (A) Representative TUNEL staining in each group (magnification, 400×). TUNEL-positive cells are stained brown. a: Control; b: Allopurinol; c: OA; d: OA+Allopurinol. (B) Quantification of the percentage of positively-stained cells in each group. (C) Representative western blot of Bax and Bcl expression in each group. GAPDH was used as a protein loading control. (D) Quantification of the Bcl and Bax protein expression levels. Data are expressed as the means $\pm \mathrm{SD}$. ${ }^{*} \mathrm{P}<0.05$ compared with the control group; ${ }^{\#} \mathrm{P}<0.05$ com-

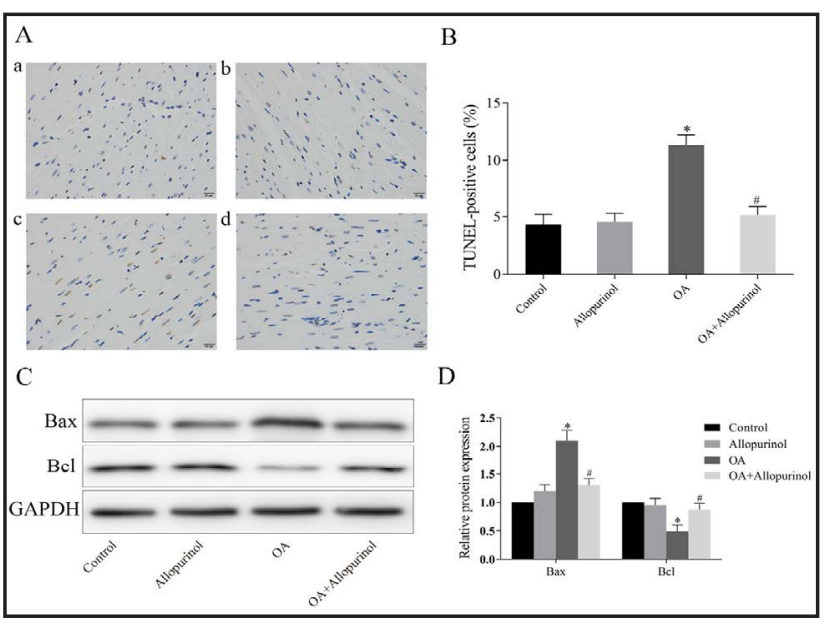
pared with the OAg.

of positive Sirius Red-stained cells (Fig. 1A, B) and increased mRNA expression levels of several fibrotic markers (collagen-I, connective tissue growth factor and transforming growth factor- $\beta$ ) (Fig. 1C). The TUNEL assay was used to evaluate cardiomyocyte apoptosis. The induction of hyperuricemia significantly increased the quantity of TUNEL-positive cells (Fig. 2A, B), increased Bax expression and decreased Bcl expression (Fig. 2C, D). However, the allopurinol pretreatment decreased the serum and urinary UA levels in groups with OA administration (Table 1) and mitigated all the above changes induced by hyperuricemia (Fig. 1 and 2).

\section{Hyperuricemia increased activation of calpain and ER stress in the rat myocardium}

After the above treatments, calpain expression and activity were tested in each group. The results revealed that calpain activity was significantly increased after OA gavage administration for 16 weeks (Fig. 3A). Calpain-1 expression but not that of calpain-2 was increased after OA administration (Fig. 3B, C). Moreover, hyperuricemia induced ER stress activation, as evidenced by the increased p-PERK, GRP78 and CHOP expression (Fig. 3D, E). However, treatment with allopurinol mitigated all the above hyperuricemia-induced changes (Fig. 3).

To demonstrate whether calpain activation occurs primarily in cardiomyocytes after $\mathrm{OA}$ treatment and whether this activation is accompanied by programmed cell death, we conducted calpain and $\alpha$-actinin immunostaining and TUNEL staining. Compared with the control group that exhibited neither calpain enrichment nor TUNEL-positive nuclei, the group with $\mathrm{OA}$ administration for 16 weeks presented with significantly increased calpain expression and TUNEL-positive staining, which was mostly located in cardiomyocytes, as 


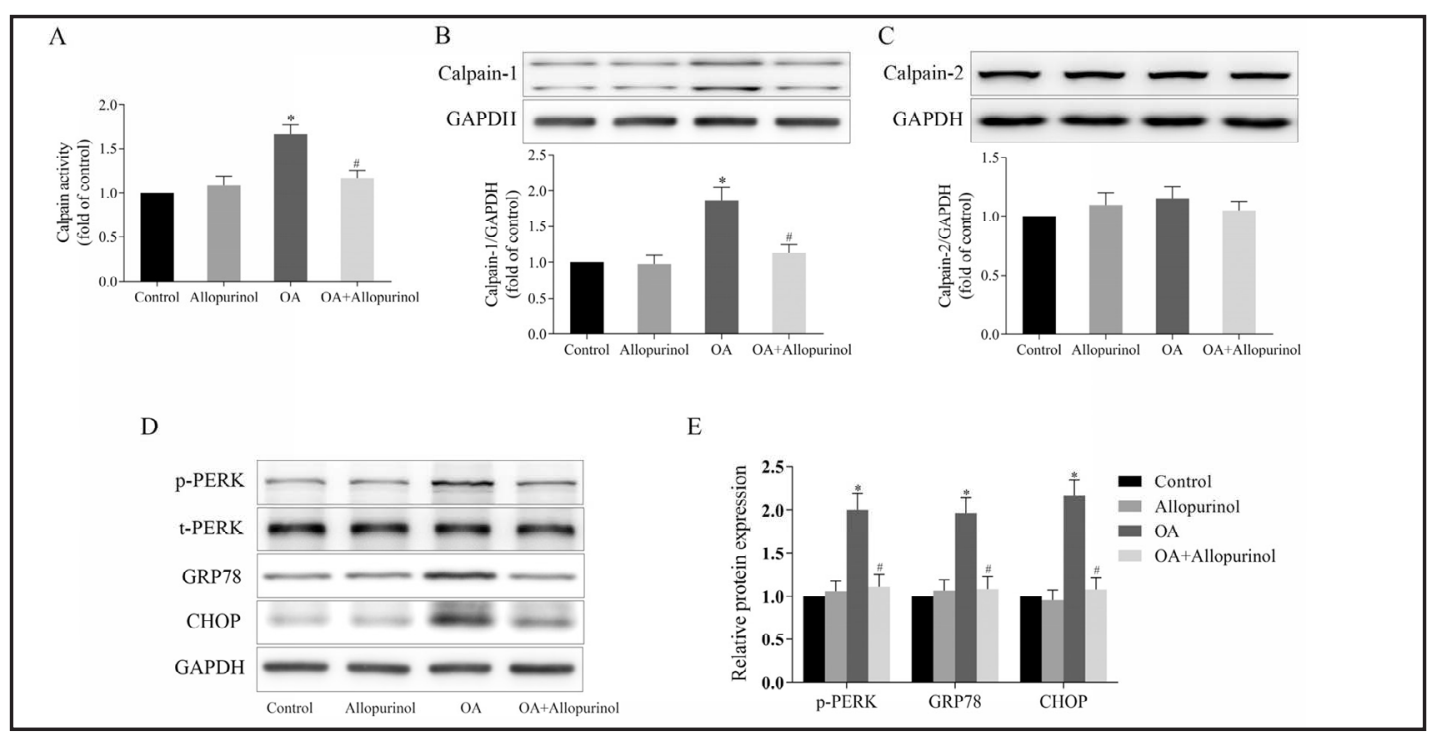

Fig. 3. Hyperuricemia increased calpain activation and ER stress in rat myocardium. (A) Calpain activity was measured in rat hearts. (B) Representative western blot and quantification of calpain-1 expression. GAPDH was used as the protein loading control. (C) Representative western blot and quantification of calpain-2 expression. (D) Representative western blots of ER stress markers (p-PERK, t-PERK, GRP78 and CHOP). (E) Quantification of the protein expression levels of ER stress markers (p-PERK, t-PERK, GRP78 and CHOP). Data are expressed as the means $\pm \mathrm{SD}$. ${ }^{*} \mathrm{P}<0.05$ compared with the control group; ${ }^{\#} \mathrm{P}<0.05$ compared with the OA group.

demonstrated by $\alpha$-actinin staining. However, these changes were prevented with the allopurinol treatment (Fig. 4).

\section{UA increased H9c2 cell apoptosis} in a dose-dependent manner

To investigate the effects of UA on cardiomyocyte survival, $\mathrm{H} 9 \mathrm{c} 2$ cells were incubated with various UA concentrations (50 to $400 \mu \mathrm{M}$ ) for 12 to $48 \mathrm{~h}$. MTT assays were used to test $\mathrm{H} 9 \mathrm{c} 2$ cell viability. As shown in Fig. 5A, during the experiments, cell viability was decreased in a time- and dose-dependent manner. After incubating with various UA doses (50 to $400 \mu \mathrm{M}$ ) for $24 \mathrm{~h}, \mathrm{H} 9 \mathrm{c} 2$ cell apoptosis was evaluated by flow cytometry using an Annexin V/PI cell apoptosis kit. As shown in Fig. 5B and 5C, the apoptosis rate of $\mathrm{H} 9 \mathrm{c} 2$ cells was increased in a dose-

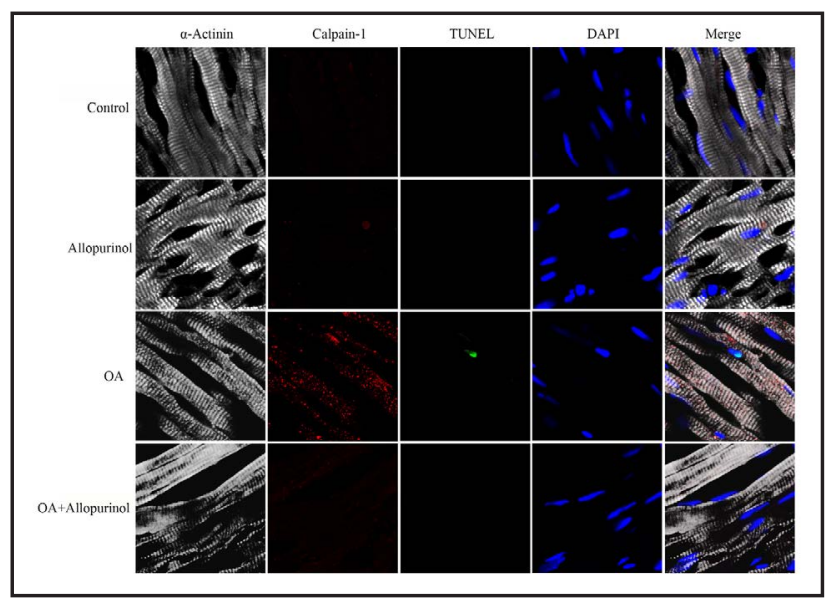

Fig. 4. Hyperuricemia increased calpain activation and cell death in the rat myocardium. Representative fluorescence confocal micrographs in each group (magnification, 400x). Fresh frozen LV tissue sections from each group were stained for $\alpha$-actinin (white) and calpain (red) expression; TdT-mediated dUTP nick-end labeling (TUNEL; green) was conducted; and the nuclei were stained blue with 4',6-diamidino-2-phenylindole (DAPI). dependent manner, with the highest apoptosis rate at the $400 \mu \mathrm{M}$ concentration.

UA increased calpain-1 expression and activity and induced ER stress in H9c2 cells

To investigate the effects of UA on calpain expression and activity in cardiomyocytes, $\mathrm{H} 9 \mathrm{c} 2$ cells were exposed to $400 \mu \mathrm{M}$ UA for various time periods $(0,3,6,12,24 \mathrm{~h})$, and 
Fig. 5. UA increased apoptosis and decreased H9c2 cell viability. H9c2 cells were treated with UA at the indicated doses $(0,50$, $100,200$ and $400 \mu \mathrm{M})$ for 12-48 h. (A) The cell survival rate was detected by MTT assay. (B) Cell apoptosis was measured using Annexin V/PI staining and flow cytometric analysis. (C) The early

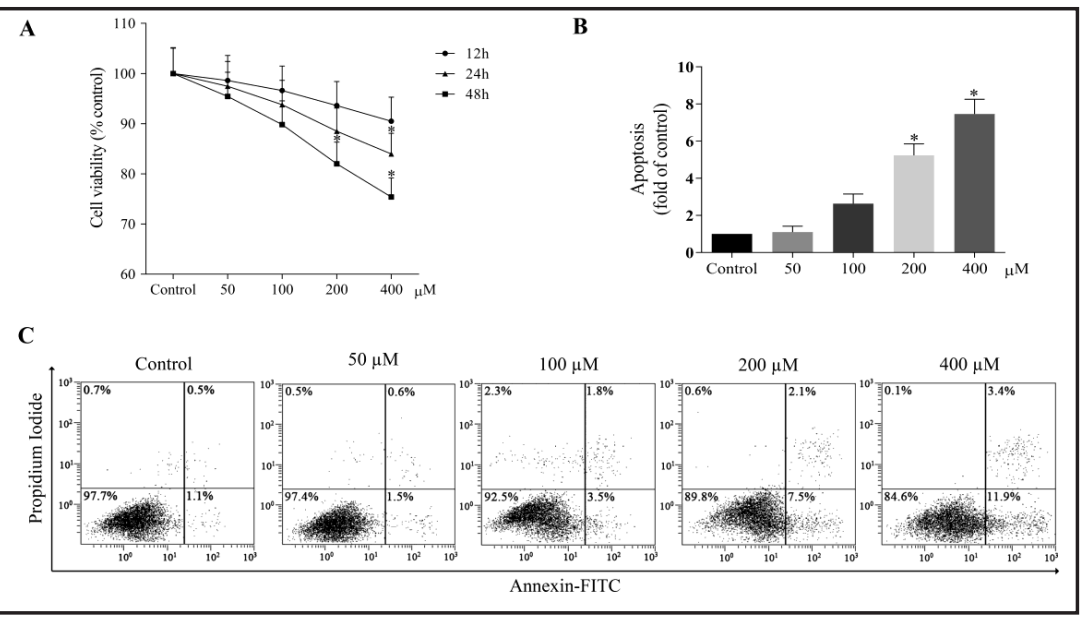
apoptosis ratio was cal-

culated. Annexin V-positive, PI-negative cells were identified as early apoptotic cells. Data are the means \pm SD. ${ }^{*} \mathrm{P}<0.05$ compared with the control group.

Fig. 6. UA increased calpain-1 expression and activity and induced endoplasmic reticulum (ER) stress in $\mathrm{H} 9 \mathrm{c} 2$ cells. $\mathrm{H} 9 \mathrm{c} 2$ cells were incubated with 400 $\mu \mathrm{M}$ UA for 3,6 , 12 and 24 h. (A) Representative western blot and quantification of calpain-1 expression. GAPDH was used as the protein loading control. (B) Representative western

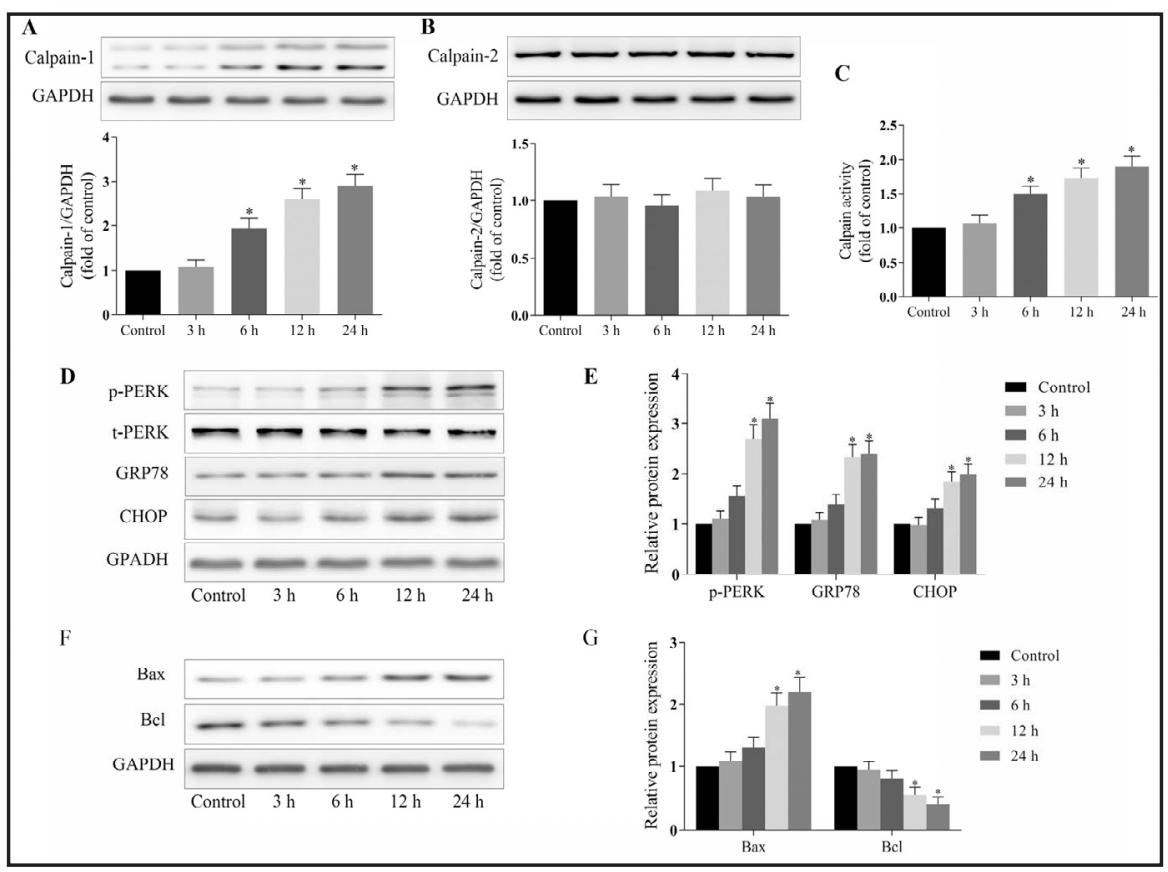
blot and quan-

tification of calpain-2 expression. (C) Calpain activity was measured in H9c2 cells. (D, E) Representative western blots and quantification of ER stress marker (p-PERK, t-PERK, GRP78 and CHOP) expression. (F, G) Representative western blots and quantification of Bax and Bcl expression. Data are the means \pm SD. ${ }^{*} \mathrm{P}<0.05$ compared with the control group.

calpain-1 and calpain-2 expression and activity were evaluated. As shown in Fig. 6A and 6B, calpain-1 protein expression, but not that of calpain-2, was significantly increased; this level started to increase at $6 \mathrm{~h}$ and continued throughout the first $24 \mathrm{~h}$. Calpain activity was also increased in a time-dependent manner (Fig. 5C).

To examine the effects of UA on ER stress in cardiomyocytes, H9c2 cells were incubated with $400 \mu \mathrm{M}$ UA for various time periods $(0,3,6,12,24 \mathrm{~h})$. As shown in Fig. 6D and 6E, p-PERK, GRP78 and CHOP expression were all increased in a time-dependent manner. Increased Bax expression and decreased Bcl expression were also observed in a similar pattern (Fig. 6F, G). 
Fig. 7. The role of calpains and ER stress in UA-induced effects in H9c2 cells. H9c2 cells were incubated with 10 $\mu \mathrm{M}$ of CI-III or $100 \mu \mathrm{M}$ of TUDCA for $30 \mathrm{~min}$ prior to administration of UA. (A) Cell apoptosis was measured using Annexin V/PI staining and flow cytometric analysis. The early apoptosis ratio was calculated. (B) Calpain activity was measured in H9c2 cells. (C, D) Representative western blots and quantification of ER stress markers ( $p$-PERK,

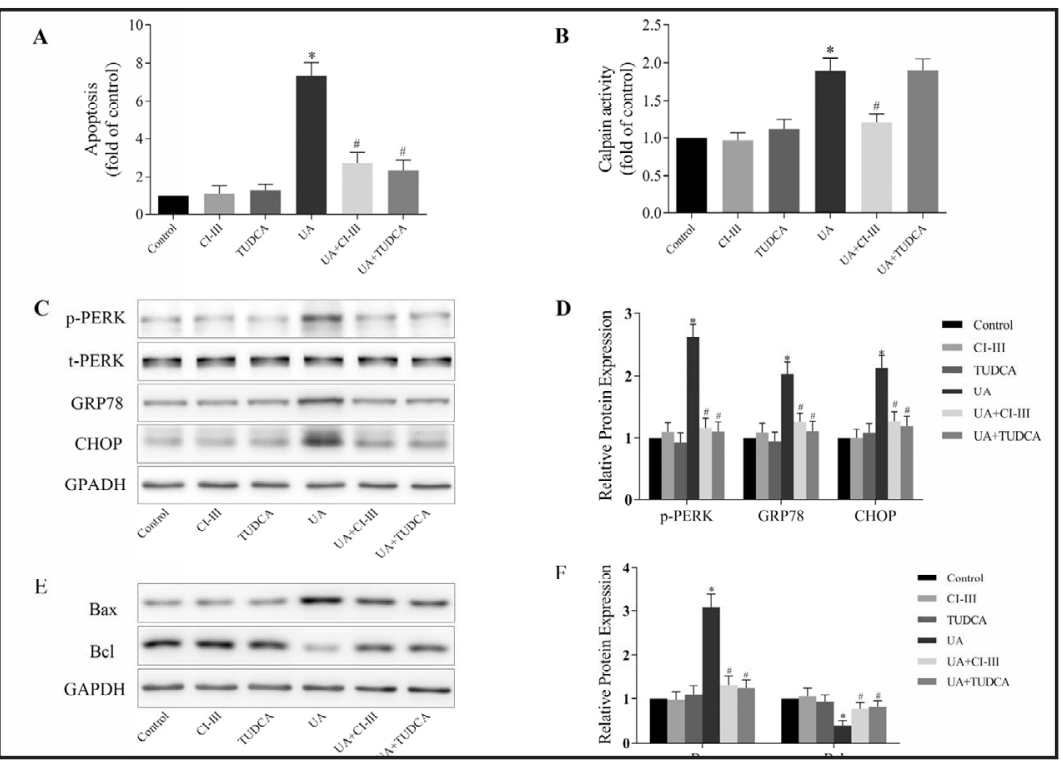
t-PERK, GRP78 and CHOP). (E, F) Representative western blots and quantification of Bax and Bcl. Data are mean $\pm \mathrm{SD} .{ }^{*} \mathrm{P}<0.05$ compared with the control group. ${ }^{*} \mathrm{P}<0.05$ compared with the UA group.

Fig. 8. Role of calpain-1 in UA-induced apoptosis and ER stress in H9c2 cells. H9c2 cells were transfected with calpain-1 siRNA or control siRNA. At $48 \mathrm{~h}$ after transfection, the cells were incubated with $400 \mu \mathrm{M}$ UA. (A) Representative western blot and quantification of calpain-1 expression. GAPDH was used as the protein loading control. (B) Calpain activity was measured in $\mathrm{H} 9 \mathrm{c} 2$ cells. (C) Cell apoptosis was measured using Annexin V/PI staining and flow cytometric analysis. The early apoptosis ratio was calculated. (D, E) Representative western blots and quantification of ER stress marker (p-PERK, t-PERK,

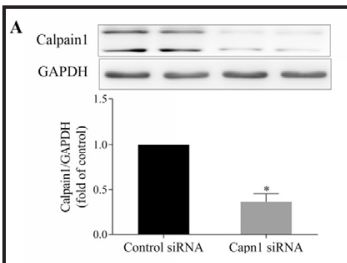

D
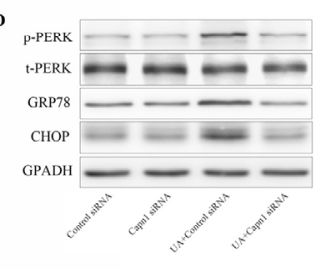

F

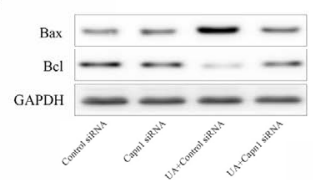

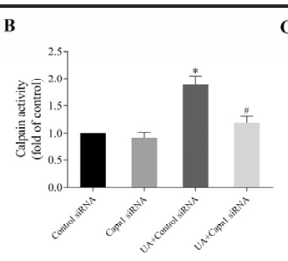

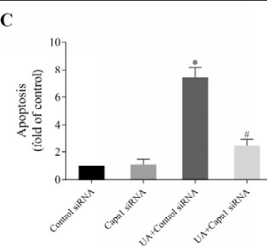

$\mathbf{E}$
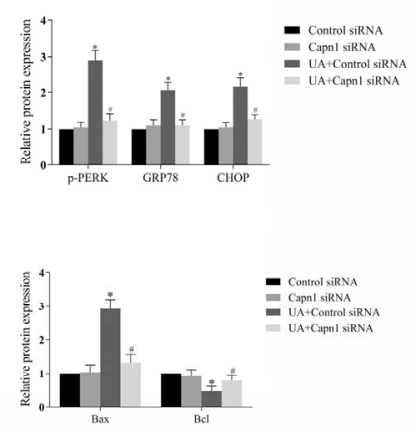
GRP78 and CHOP) expression. (F, G)

Representative western blots and quantification of Bax and Bcl expression. Data are the means \pm SD. ${ }^{*} \mathrm{P}<0.05$ compared with the control siRNA group. ${ }^{\#} \mathrm{P}<0.05$ compared with the UA+control siRNA.

The role of calpains and ER stress in the UA-induced effects in H9c2 cells

To investigate the roles of calpain and ER stress in UA-induced apoptosis, H9c2 cells were pretreated with the calpain inhibitor III (CI-III) or ER stress inhibitor tauroursodeoxycholic acid (TUDCA) for 30 minutes before incubating with UA. As shown in Fig. 7, the CI-III pretreatment significantly mitigated the UA-induced effects on calpain expression, ER stress and cellular apoptosis. TUDCA also alleviated UA-induced ER stress and apoptosis but did not alter calpain activity in H9c2 cells.

To confirm the role of calpain-1 in UA-induced ER stress and apoptosis, H9c2 cells were transfected with calpain-1 siRNA. Calpain-1 siRNA significantly decreased calpain-1 expression (Fig. 8A), suggesting that the knockdown was successful. Compared with the 


\section{Cellular Physiology Cell Physiol Biochem 2018;45:2122-2135 \\ \begin{tabular}{ll|l} 
DOI: 10.1159/000488048 & and Biochemistry Published online:March 12, 2018 & $\begin{array}{l}\text { O } 2018 \text { The Author(s). Published by S. Karger AG, Basel } \\
\text { www.karger.com/cpb }\end{array}$
\end{tabular}}

Yan et al.: UA Induces Apoptosis in Cardiomyocytes via Calpain-1

scramble siRNA transfection, the administration of UA induced calpain activation (Fig. 8B) and ER stress (Fig. 8D, E), as evidenced by the increased expression of ER stress markers (p-PEFK, GRP78, CHOP), and decreased cellular apoptosis (Fig. 8C, F, G), as evidenced by the increased Bax expression and decreased Bcl expression. However, the calpain-1 siRNA transfection alleviated the UA-induced ER stress and cellular apoptosis.

\section{Discussion}

The aim of the current study was to test the effects of UA on cardiomyocyte survival and cardiac function and to investigate the roles of calpains and ER stress in UA-induced adverse effects on the heart. The major findings were as follows: 1. UA accumulation resulted in cardiomyocyte death and impaired diastolic function in rat hearts. 2. UA induced ER stress and subsequent apoptosis through calpain-1 activation.

UA is the end metabolite of purine metabolism [15], and the serum UA level is significantly higher in humans than in other mammals due to the loss of uricase activity [16,17]. High physiological UA levels in humans and higher primates have been hypothesized to be protective against oxidative stress $[18,19]$. However, a series of well-grounded epidemiological studies have demonstrated the association between high serum UA levels and increased risk for a variety of diseases [20-23]. In the cardiovascular system, hyperuricemia is associated with an increased risk for and/or worse outcome in coronary heart disease [4, 24], heart failure [3, 25], hypertension [26, 27], atrial fibrillation [28, 29] and general cardiovascular events [30]. Despite these associations, some have argued that serum UA is more of a marker rather than a contributor to cardiovascular disease [31]. To answer that question, animal studies with homogenous baseline and manipulated serum UA levels are needed. Chen et al. reported that hyperuricemic infarcted rats (induced by $\mathrm{OA}$ ) presented with more severe LV dysfunction and myocardial hypertrophy compared with normouricemic infarcted rats [7]. In the present study, hyperuricemia was also induced by OA gavage administration in SpragueDawley rats. The results revealed that hyperuricemia resulted in impaired diastolic function in unstressed rat hearts and did not significantly influence serum creatinine levels, which was accompanied by increased apoptosis and interstitial fibrosis, suggesting hyperuricemia causes diastolic dysfunction in unstressed rat hearts via increasing cardiomyocyte apoptosis and interstitial fibrosis. In contrast, pretreatment with allopurinol decreased the serum UA level and alleviated hyperuricemia-induced apoptosis, fibrosis and diastolic dysfunction, suggesting that hyperuricemia, rather than the nonspecific effects of $\mathrm{OA}$, is responsible for these adverse effects, which is generally consistent with the findings of previous studies [7, 8]. To confirm these findings in vitro, cultured $\mathrm{H} 9 \mathrm{c} 2$ cells were incubated with various UA doses, and the results revealed that the UA treatment dose-dependently increased apoptosis and decreased cell viability.

In previous studies, the unfavorable effects of UA on the cardiovascular system were attributed to its roles in several pathological processes such as inflammation, myocardial oxidative stress, insulin resistance, and interstitial fibrosis [6-8]. However, the underlying mechanisms remain largely elusive. Calpains belong to a family of cytosolic calcium-dependent cysteine proteases and are involved in a broad range of cellular functions [11]. Among the 15 gene reported products in the mammalian calpain family, calpain- 1 and calpain- 2 are the two major isoforms, are ubiquitously expressed and have been well studied. Both calpain-1 and calpain-2 are expressed in the heart [32]. In a series of recent publications, calpains have been reported to participate in several conditions affecting the cardiovascular system, such as hypertension [12], atherosclerosis [33], myocardial infarction [13], and heart failure [14]. We wondered if calpains are involved in the UA-induced effects in the heart. In the current study, calpain-1, not calpain-2, expression and activation were increased in the cardiac tissue of hyperuricemic rats, and these changes were abolished by the allopurinol treatment. Similar results were also observed in cultured H9c2 cells with direct UA administration. To confirm the role of calpain-1 in this process, calpain- 1 expression was knocked down by 
calpain-1 siRNA transfection in cultured H9c2 before the treatment with UA. The results revealed that calpain-1 knockdown significantly alleviated UA-induced apoptosis. These findings demonstrated that UA induces apoptosis in cardiomyocytes through increasing calpain-1 expression and activation, which to our knowledge, has not been reported before. However, with the current evidence, we cannot be certain whether the increased calpain-1 activity is solely due to the increased expression, or if UA influences calpain-1 activation, which can be investigated in future studies.

The ER is the site of protein synthesis and processing and is exquisitely sensitive to cell homeostasis alterations. The dysregulation of protein synthesis/processing within the ER causes the accumulation of unfolded protein, triggering the unfolded protein response (UPR), which reduces the accumulation of unfolded protein by increasing the level of ER resident chaperones, such as 78-kDa glucose-regulated protein (GRP78) [34], inhibiting protein translation, and accelerating the clearance of unfolded proteins. However, if the stress exceeds the capacity of these adaptive mechanisms, the ER initiates apoptotic signaling, which may lead to cell death. PERK is an ER membrane transducer that mediates UPR activation. When UPR cannot restore ER homeostasis, PERK activation leads to eIF2 $\alpha$ phosphorylation, which selectively induces a transcription factor called ATF4. ATF4 enhances the expression of CCAAT/enhancer-binding protein homologous protein (CHOP), which exerts pro-apoptotic effects $[35,36]$. In several cell lines, ER stress has been found to be activated following UA exposure [37-39] and was reported to be involved in UA-induced apoptosis in one of these studies [39]. The results of the present study revealed that high levels of UA induced ER stress in cultured cardiomyocytes as well as rat myocardium, as evidenced by the increased GRP78, p-PERK, and CHOP expression, which are widely used as markers of ER stress [40-43].

Tauroursodeoxycholic acid (TUDCA) is a hydrophilic nontoxic derivative of endogenous bile acids [44]. As a commonly used inhibitor of ER stress [45-47], TUDCA has been reported to exert preventive effects on ER stress during the early stage of apoptosis before mitochondrial involvement $[44,48]$. In the present study, administration of the ER stress inhibitor TUDCA reduced the expression of the ER stress markers GRP78, p-PERK, and CHOP and attenuated UA-induced apoptosis in H9c2 cells, suggesting that ER stress participates in UA-induced apoptosis. However, the relationship between ER stress and calpain-1 in the UA-induced effects in cardiomyocytes is unknown. In a previous study, Zheng et al. reported that calpain-1 up-regulation induced ER stress and JNK1/2 activation, thereby mediating cardiomyocyte apoptosis [49]. In the present study, pretreating with the calpain inhibitor CIIII or with calpain-1 siRNA mitigated the UA-induced ER stress and subsequent apoptosis, while pretreating with the ER stress inhibitor TUDCA did not significantly influence calpain activity, indicating that calpain-1 is an upstream regulator of ER stress in the UA-induced effects in cardiomyocytes (Fig. 9).

\section{Conclusion}

In summary, the present study revealed that high UA

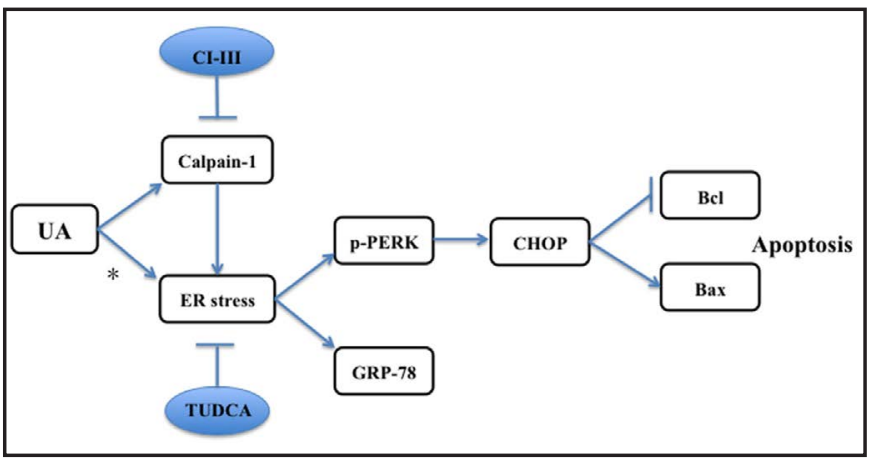

Fig. 9. Schematic diagram illustrating the mechanisms by which UA induces apoptosis in cardiomyocytes. Pretreatment with the calpain inhibitor CI-III mitigated UA-induced ER stress and subsequent apoptosis, while pretreatment with the ER stress inhibitor TUDCA did not significantly influence calpain-1 activity, indicating that UA induces cardiomyocyte apoptosis through activation of calpain- 1 and ER stress and that calpain- 1 is an upstream regulator of ER stress in the UA-induced effects in cardiomyocytes. *Other mechanisms of UA-induced ER stress have also reported $[37,38]$. 
levels resulted in impaired diastolic function in rat heart, which was accompanied by increased cardiomyocyte apoptosis and interstitial fibrosis. Furthermore, we demonstrated that UA induced cardiomyocyte ER stress and subsequent apoptosis through calpain-1 activation. However, the mechanisms by which UA increased calpain-1 expression and activation were not investigated in this current study and is a topic for our on-going studies. These results may provide new insights into the mechanisms underlying the association between hyperuricemia and increased cardiovascular risk and hopefully identify new treatment targets.

\section{Acknowledgements}

This study was supported by the National Natural Science Foundation of China (No. 81571363) and the grant from the Scientific Research Fund of Shanghai Jiao Tong University Affiliated Sixth People's Hospital to Dr. KanKai Chen.

\section{Disclosure Statement}

The authors declare no conflicts of interest.

\section{References}

1 Mandal AK, Mount DB: The molecular physiology of uric acid homeostasis. Annu Rev Physiol 2015;77:323345 .

2 Johnson RJ: Why focus on uric acid? Curr Med Res Opin 2015;31:S3-7.

3 Huang H, Huang B, Li Y, Huang Y, Li J, Yao H, Jing X, Chen J, Wang J: Uric acid and risk of heart failure: A systematic review and meta-analysis. Eur J Heart Fail 2014;16:15-24.

4 Li M, Hu X, Fan Y, Li K, Zhang X, Hou W, Tang Z: Hyperuricemia and the risk for coronary heart disease morbidity and mortality a systematic review and dose-response meta-analysis. Sci Rep 2016;6:19520.

5 Braga F, Pasqualetti S, Ferraro S, Panteghini M: Hyperuricemia as risk factor for coronary heart disease incidence and mortality in the general population: A systematic review and meta-analysis. Clin Chem Lab Med 2016;54:7-15.

6 Kanellis J, Kang DH: Uric acid as a mediator of endothelial dysfunction, inflammation, and vascular disease. Semin Nephrol 2005;25:39-42.

7 Chen CC, Hsu YJ, Lee TM: Impact of elevated uric acid on ventricular remodeling in infarcted rats with experimental hyperuricemia. Am J Physiol Heart Circ Physiol 2011;301:H1107-1117.

8 Jia G, Habibi J, Bostick BP, Ma L, DeMarco VG, Aroor AR, Hayden MR, Whaley-Connell AT, Sowers JR: Uric acid promotes left ventricular diastolic dysfunction in mice fed a western diet. Hypertension 2015;65:531539.

-9 Ono Y, Sorimachi H: Calpains: An elaborate proteolytic system. Biochim Biophys Acta 2012;1824:224-236.

10 Campbell RL, Davies PL: Structure-function relationships in calpains. Biochem J 2012;447:335-351.

-11 Goll DE, Thompson VF, Li H, Wei W, Cong J: The calpain system. Physiol Rev 2003;83:731-801.

$>12$ Averna M, De Tullio R, Salamino F, Minafra R, Pontremoli S, Melloni E: Age-dependent degradation of calpastatin in kidney of hypertensive rats. J Biol Chem 2001;276:38426-38432.

13 Mani SK, Balasubramanian S, Zavadzkas JA, Jeffords LB, Rivers WT, Zile MR, Mukherjee R, Spinale FG, Kuppuswamy D: Calpain inhibition preserves myocardial structure and function following myocardial infarction. Am J Physiol Heart Circ Physiol 2009;297:H1744-1751.

14 Letavernier E, Zafrani L, Perez J, Letavernier B, Haymann JP, Baud L: The role of calpains in myocardial remodelling and heart failure. Cardiovasc Res 2012;96:38-45.

15 Richette P, Bardin T: Gout. Lancet 2010;375:318-328.

16 Alvarez-Lario B, Macarron-Vicente J: Uric acid and evolution. Rheumatology (Oxford) 2010;49:2010-2015. 


\section{Cellular Physiology Cell Physiol Biochem 2018;45:2122-2135 \begin{tabular}{c|c|c|}
\cline { 2 - 3 } DOI: 10.1159/000488048 & $\begin{array}{l}\text { O 2018 The Author(s). Published by S. Karger AG, Basel } \\
\text { www.karger.com/cpb }\end{array}$
\end{tabular} and Biochemistry}

17 Maiuolo J, Oppedisano F, Gratteri S, Muscoli C, Mollace V: Regulation of uric acid metabolism and excretion. Int J Cardiol 2016;213:8-14.

18 Glantzounis GK, Tsimoyiannis EC, Kappas AM, Galaris DA: Uric acid and oxidative stress. Curr Pharm Des 2005;11:4145-4151.

19 Alvarez-Lario B, Macarron-Vicente J: Is there anything good in uric acid? QJM 2011;104:1015-1024.

20 Forman JP, Choi H, Curhan GC: Uric acid and insulin sensitivity and risk of incident hypertension. Arch Intern Med 2009;169:155-162.

-21 Nakanishi N, Okamoto M, Yoshida H, Matsuo Y, Suzuki K, Tatara K: Serum uric acid and risk for development of hypertension and impaired fasting glucose or type ii diabetes in japanese male office workers. Eur J Epidemiol 2003;18:523-530. Maharani N, Kuwabara M, Hisatome I: Hyperuricemia and atrial fibrillation. Int Heart J 2016;57:395-399. Ekundayo OJ, Dell'Italia LJ, Sanders PW, Arnett D, Aban I, Love TE, Filippatos G, Anker SD, Lloyd-Jones DM, Bakris G, Mujib M, Ahmed A: Association between hyperuricemia and incident heart failure among older adults: A propensity-matched study. Int J Cardiol 2010;142:279-287.

24 von Lueder TG, Girerd N, Atar D, Agewall S, Lamiral Z, Kanbay M, Pitt B, Dickstein K, Zannad F, Rossignol P: Serum uric acid is associated with mortality and heart failure hospitalizations in patients with complicated myocardial infarction: Findings from the high-risk myocardial infarction database initiative. Eur J Heart Fail 2015;17:1144-1151.

-25 Vaduganathan M, Greene SJ, Ambrosy AP, Mentz RJ, Subacius HP, Chioncel O, Maggioni AP, Swedberg K, Zannad F, Konstam MA, Senni M, Givertz MM, Butler J, Gheorghiade M: Relation of serum uric acid levels and outcomes among patients hospitalized for worsening heart failure with reduced ejection fraction (from the efficacy of vasopressin antagonism in heart failure outcome study with tolvaptan trial). Am J Cardiol 2014;114:1713-1721.

-26 Kuwabara M, Niwa K, Nishi Y, Mizuno A, Asano T, Masuda K, Komatsu I, Yamazoe M, Takahashi O, Hisatome I: Relationship between serum uric acid levels and hypertension among japanese individuals not treated for hyperuricemia and hypertension. Hypertens Res 2014;37:785-789.

27 Sun HL, Pei D, Lue KH, Chen YL: Uric acid levels can predict metabolic syndrome and hypertension in adolescents: A 10-year longitudinal study. PLoS One 2015;10:e0143786.

28 Chao TF, Hung CL, Chen SJ, Wang KL, Chen TJ, Lin YJ, Chang SL, Lo LW, Hu YF, Tuan TC, Chen SA: The association between hyperuricemia, left atrial size and new-onset atrial fibrillation. Int J Cardiol 2013;168:4027-4032.

29 Kawasoe S, Kubozono T, Yoshifuku S, Ojima S, Oketani N, Miyata M, Miyahara H, Maenohara S, Ohishi M: Uric acid level and prevalence of atrial fibrillation in a japanese general population of 285, 882 Circ J 2016;80:2453-2459.

-30 Kleber ME, Delgado G, Grammer TB, Silbernagel G, Huang J, Kramer BK, Ritz E, Marz W: Uric acid and cardiovascular events: A mendelian randomization study. J Am Soc Nephrol 2015;26:2831-2838.

-31 Caliskan M, Guven A, Ciftci O, Ozulku M, Gunday M, Barutcu I: Serum uric acid and carotid artery intima media thickness in patients with masked hypertension. Acta Cardiol 2014;69:417-423.

-32 Sorimachi H, Ono Y: Regulation and physiological roles of the calpain system in muscular disorders. Cardiovasc Res 2012;96:11-22.

-33 Miyazaki T, Taketomi Y, Takimoto M, Lei XF, Arita S, Kim-Kaneyama JR, Arata S, Ohata H, Ota H, Murakami M, Miyazaki A: M-calpain induction in vascular endothelial cells on human and mouse atheromas and its roles in ve-cadherin disorganization and atherosclerosis. Circulation 2011;124:2522-2532.

-34 Dudek J, Benedix J, Cappel S, Greiner M, Jalal C, Muller L, Zimmermann R: Functions and pathologies of bip and its interaction partners. Cell Mol Life Sci 2009;66:1556-1569.

-35 Tabas I, Ron D: Integrating the mechanisms of apoptosis induced by endoplasmic reticulum stress. Nat Cell Biol 2011;13:184-190.

-36 Gerbino A, Bottillo I, Milano S, Lipari M, Zio R, Morlino S, Mola MG, Procino G, Re F, Zachara E, Grammatico P, Svelto M, Carmosino M: Functional characterization of a novel truncating mutation in lamin a/c gene in a family with a severe cardiomyopathy with conduction defects. Cell Physiol Biochem 2017;44:1559-1577.

-37 Li S, Zhao F, Cheng S, Wang X, Hao Y: Uric acid-induced endoplasmic reticulum stress triggers phenotypic change in rat glomerular mesangial cells. Nephrology (Carlton) 2013;18:682-689. 


\section{Cellular Physiology Cell Physiol Biochem 2018;45:2122-2135

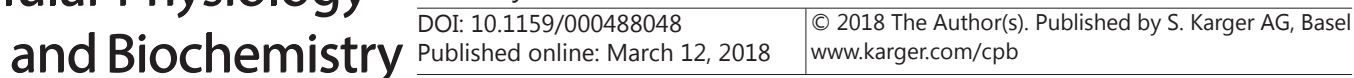

Yan et al.: UA Induces Apoptosis in Cardiomyocytes via Calpain-1

-38 Choi YJ, Shin HS, Choi HS, Park JW, Jo I, Oh ES, Lee KY, Lee BH, Johnson RJ, Kang DH: Uric acid induces fat accumulation via generation of endoplasmic reticulum stress and srebp-1c activation in hepatocytes. Lab Invest 2014;94:1114-1125.

39 Li P, Zhang L, Zhang M, Zhou C, Lin N: Uric acid enhances pkc-dependent enos phosphorylation and mediates cellular er stress: A mechanism for uric acid-induced endothelial dysfunction. Int J Mol Med 2016;37:989-997.

-40 Kennedy D, Samali A, Jager R: Methods for studying er stress and upr markers in human cells. Methods Mol Biol 2015;1292:3-18.

-41 Wang C, Wu M, Arvapalli R, Dai X, Mahmood M, Driscoll H, Rice KM, Blough E: Acetaminophen attenuates obesity-related renal injury through er-mediated stress mechanisms. Cell Physiol Biochem 2014;33:11391148.

42 Lin Y, Zhu J, Zhang X, Wang J, Xiao W, Li B, Jin L, Lian J, Zhou L, Liu J: Inhibition of cardiomyocytes hypertrophy by resveratrol is associated with amelioration of endoplasmic reticulum stress. Cell Physiol Biochem 2016;39:780-789.

43 Zhang N, Wei WY, Yang Z, Che Y, Jin YG, Liao HH, Wang SS, Deng W, Tang QZ: Nobiletin, a polymethoxy flavonoid, protects against cardiac hypertrophy induced by pressure-overload via inhibition of napdh oxidases and endoplasmic reticulum stress. Cell Physiol Biochem 2017;42:1313-1325.

44 Xie Q, Khaoustov VI, Chung CC, Sohn J, Krishnan B, Lewis DE, Yoffe B: Effect of tauroursodeoxycholic acid on endoplasmic reticulum stress-induced caspase-12 activation. Hepatology 2002;36:592-601.

45 Ozcan L, Ergin AS, Lu A, Chung J, Sarkar S, Nie D, Myers MG, Jr., Ozcan U: Endoplasmic reticulum stress plays a central role in development of leptin resistance. Cell metabolism 2009;9:35-51.

-46 Ge W, Zhang Y, Han X, Ren J: Cardiac-specific overexpression of catalase attenuates paraquat-induced myocardial geometric and contractile alteration: Role of er stress. Free Radic Biol Med 2010;49:2068-2077.

-47 Ding W, Wang B, Zhang M, Gu Y: Involvement of endoplasmic reticulum stress in uremic cardiomyopathy: Protective effects of tauroursodeoxycholic acid. Cell Physiol Biochem 2016;38:141-152.

48 Ozcan U, Yilmaz E, Ozcan L, Furuhashi M, Vaillancourt E, Smith RO, Gorgun CZ, Hotamisligil GS: Chemical chaperones reduce er stress and restore glucose homeostasis in a mouse model of type 2 diabetes. Science 2006;313:1137-1140.

49 Zheng D, Wang G, Li S, Fan GC, Peng T: Calpain-1 induces endoplasmic reticulum stress in promoting cardiomyocyte apoptosis following hypoxia/reoxygenation. Biochim Biophys Acta 2015;1852:882-892. 